\title{
Cortical Hyper-Connectivity in a Stroke Patient with Rotated Drawing
}

\author{
Valentina Sebastiani ${ }^{a} \quad$ Piero Chiacchiaretta $^{a}$ Luigi Pavone $^{b}$ \\ Antonio Sparano $^{b}$ Giovanni Grillea ${ }^{b}$ Sara Spadone ${ }^{a}$ Paolo Capotosto ${ }^{a}$ \\ Giorgia Committeri $^{a}$ Antonello Baldassarre ${ }^{a}$ \\ aDepartment of Neuroscience, Imaging and Clinical Sciences - University of Chieti-Pescara,

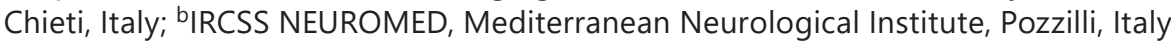

\section{Keywords}

Stroke $\cdot$ Rotated drawing $\cdot$ Functional connectivity $\cdot$ Brain networks

\begin{abstract}
In the present case report, we investigated the cortical networks of a patient (DDA) affected by right parietal stroke who showed a constructional phenomenon, in which when coping and recalling from memory a complex figure, the model was reproduced rotated of $90^{\circ}$ along the vertical axis. Previous studies suggested that rotation on copy is associated with visuospatial impairments and abnormalities in parietal cortex, whereas rotation on recall might be related to executive deficits and dysfunction of frontal regions. Here, we computed the DDA's restingstate functional connectivity $(F C)$ derived from cortical regions of the dorsal attention (DAN) and the frontal portion of the executive-control network ( $\mathrm{FCCN}$ ), which are involved in the control of visuospatial attention and multiple executive functions, respectively. We observed that, as compared to a control group of right stroke patients without drawing rotation, DDA exhibited selective increased FC of the DAN and $\mathrm{FECN}$, but not of task-irrelevant language network, within the undamaged hemisphere. These patterns might reflect a pathological communication in such networks leading to impaired attentional and executive operations required to reproduce the model in the correct orientation. Notably, such enhancement of FC was not detected in a patient with a comparable neuropsychological profile as DDA, yet without rotated drawing, suggesting that network-specific modulations in DDA might be ascribed to the constructional phenomenon of rotated drawing.
\end{abstract}

\section{Karger $\stackrel{2}{\circ}$}




\section{Introduction}

Rotated drawing is a constructional phenomenon in which when coping or recalling from memory a complex figure, the model is reproduced rotated of $\pm 90^{\circ}$ or $180^{\circ}$, although spatial relationships among basic elements are preserved [1]. Rotation has been reported in several clinical cohorts such as neurodegenerative [2] and stroke [3] patients, with a slightly higher frequency after right hemisphere lesion. Previous neuroimaging findings $[2,3]$ indicated that rotation on copy is related to dysfunctional activity in parietal regions belonging to the so-called dorsal attention network (DAN) involved in control of the visuospatial attention [4]. Based on neuropsychological evidence, it has also been suggested that rotation on recall might be associated with executive deficits. Yet the neural correlates of such constructional phenomenon are still unclear. In the current case report, we investigated, for the first time to our knowledge, the functional brain networks associated with rotated drawing after stroke. Specifically, we describe the neuropsychological profile and the patterns of altered resting-state functional connectivity (FC) by means of functional MRI [5], in a patient (DDA) showing rotation of drawings after a focal lesion to the right parietal cortex (Fig. 1). On the light of the studies mentioned above, we investigated the pathological resting-state FC within the DAN as well as the frontal portion of the executive-control network (fECN) [6] also known as frontoparietal network [7], which is associated with several executive functions including working memory, control processes, and set shifting.

\section{Case Report}

\section{Case Presentation}

A 42-year-old right-handed male (DDA, 13-year of education), factory worker, presented at the Stroke Unit of the NEUROMED hospital (Pozzilli, Italy) in April 2017 with neck headache and right retro-auricular pain. Medical records indicated no neurological/ psychiatric or alcohol/drug medical history. Furthermore, the patient was not on medication/pharmacological treatment before the stroke onset. CT and RM scans delineated the presence of parietal ischemic stroke in the right hemisphere. At the neurological examination, DDA was wakeful, spatially, and temporally oriented with a fluent speech. A week after the stroke onset, the patient underwent a neuropsychological evaluation (see Material and Methods) in which we observed a distinctive constructional phenomenon: DDA indeed exhibited rotation through $90^{\circ}$ along the vertical axis of the Rey-Osterrieth

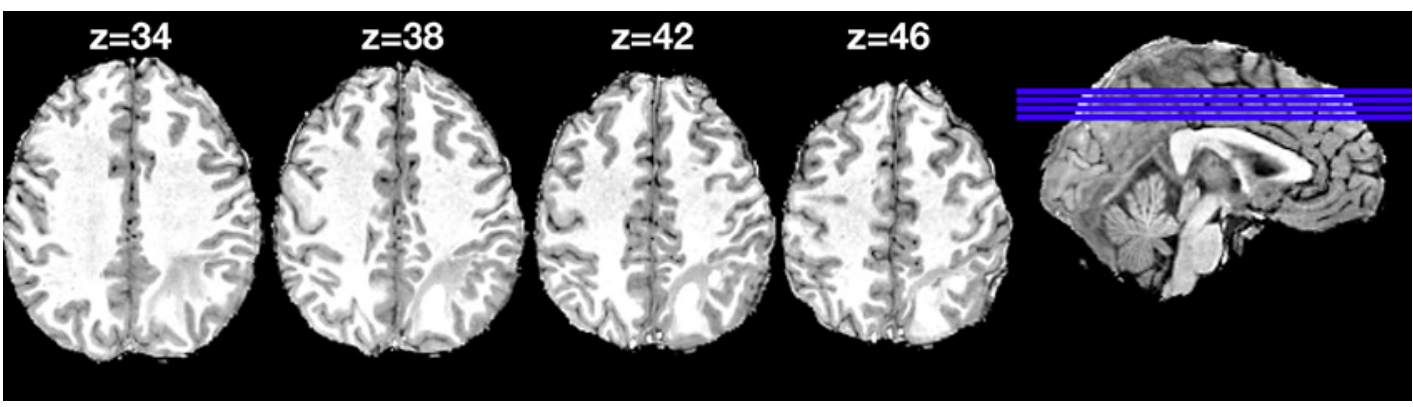

Fig. 1. The figure displays multiple axial slices of T1-weighted MRI of patient DDA. Z-coordinates are in Talairach space. 


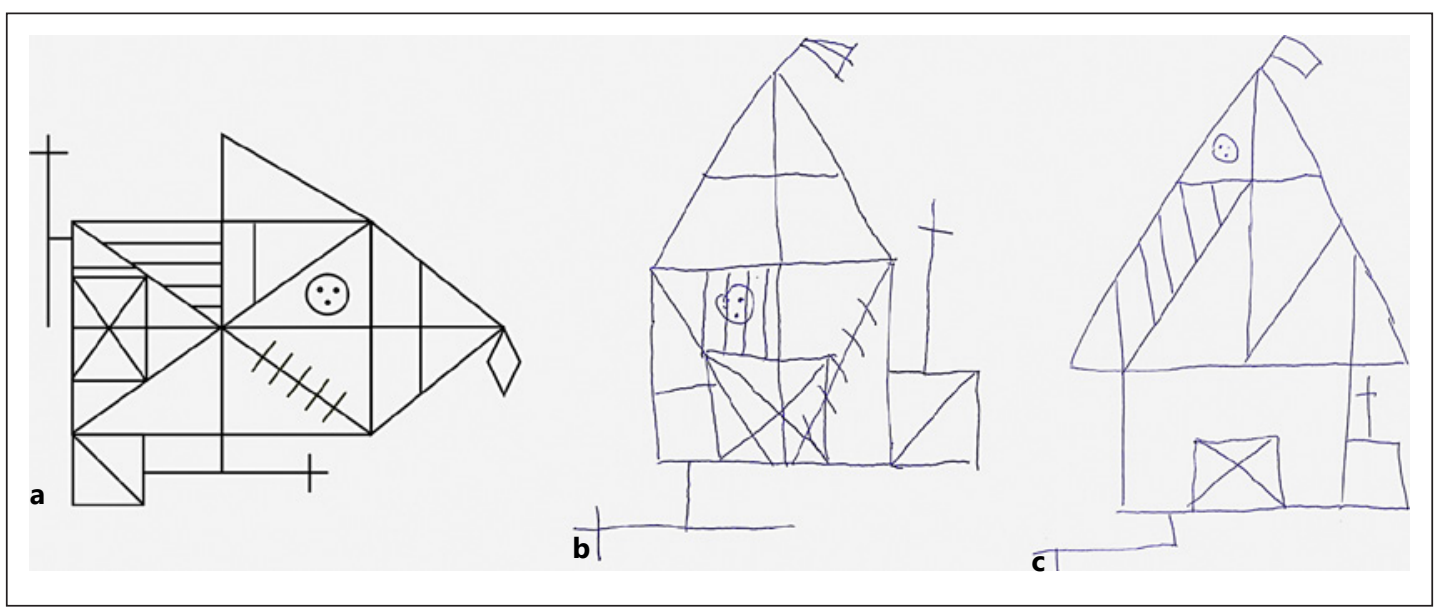

Fig. 2. Rotated copy (B) and recall (C) of the Rey-Osterrieth complex figure (A).

Complex Figure (ROCF) on both copy and recall after 10 min (Fig. 2). Furthermore, DDA was submitted to structural and resting-state functional MRI session at the same day of neuropsychological evaluation.

\section{Material and Methods}

\section{Neuropsychological Assessment}

Patient DDA and a control group of 11 right hemisphere damaged patients ( $5 \mathrm{~F}$, mean age 69 years, SD = 11 years, $<2$ weeks from stroke onset) underwent a neuropsychological assessment of the main cognitive domains including General Cognitive Efficiency; Praxis Abilities; Short- and Long-Term Memory (Verbal and Spatial); Visuospatial Attention and Executive Functions (see Table 1 for DDA's raw scores).

\section{fMRI Scanning}

DDA and a control group of 11 right hemisphere damaged patients (5 F, mean age 69 years, SD = 11 years, $<2$ weeks from stroke onset) who did not exhibit rotated drawing, underwent a structural and functional MRI session. Written informed consent was obtained from all participants of the study. The research was approved by the Institutional Review Board of NEUROMED. MRI scanning was performed with GE Signa HDxt 3T at NEUROMED within $24 \mathrm{~h}$ of the neuropsychological assessment. Structural scans consisted of: (1) an axial T1-weighted 3D SPGR (TR $=1,644 \mathrm{~ms}, \mathrm{TE}=2.856 \mathrm{~ms}$, flip angle $=13^{\circ}$, voxel size $=$ $1.0 \times 1.0 \times 1.0 \mathrm{~mm}$ ) and (2) an axial T2-weighted turbo spin-echo $(\mathrm{TR}=2.856 \mathrm{~ms}, \mathrm{TE}=$ $127.712 \mathrm{~ms}$, slice thickness $3 \mathrm{~mm}$, matrix size:512 $\times 512$ ). Resting-state functional scans were acquired with a gradient echo EPI sequence $(\mathrm{TR}=1,714 \mathrm{~ms}, \mathrm{TE}=30 \mathrm{~ms}, 34$ contiguous $3.6 \mathrm{~mm}$ slices), during which participants were instructed to keep open eyes in a low luminance environment. Three 7-min resting-state fMRI runs, each including 128 vol, were acquired.

\section{fMRI Preprocessing and Quality Control of Resting-State fMRI Data}

Functional MRI data underwent several preprocessing steps as reported in our previous study [8]. Prior to FC mapping, motion contaminated frames were identified and removed using the metric DVARS which is the root mean square change of the temporally differentiated 
Case Reports in

Neurology

\begin{tabular}{l}
\hline Case Rep Neurol 2021;13:677-686 \\
\hline DOI: 10.1159/000518844
\end{tabular} \begin{tabular}{l}
$\begin{array}{l}\text { ○ 2021 The Author(s). Published by S. Karger AG, Basel } \\
\text { www.karger.com/crn }\end{array}$ \\
\hline
\end{tabular}

Sebastiani et al.: Brain Networks and Rotated Drawing after Stroke

Table 1. Neuropsychological profile of patient DDA

\begin{tabular}{|c|c|}
\hline Neuropsychological battery & Raw scores \\
\hline \multicolumn{2}{|l|}{ General cognitive efficiency } \\
\hline Mini mental state examination & $27 / 30$ \\
\hline Clock drawing test & $9,5 / 13$ \\
\hline \multicolumn{2}{|l|}{ Praxis abilities } \\
\hline Copying geometrical figures & $13 / 14$ \\
\hline Copy of Rey-Osterrieth complex figure & $13 / 36^{*}$ \\
\hline \multicolumn{2}{|l|}{ Memory } \\
\hline Word span & $4 / 10$ \\
\hline Corsi's block tapping test & $4 / 10^{*}$ \\
\hline Story recall memory test & $13,5 / 16$ \\
\hline \multicolumn{2}{|l|}{ Rey 15 -item memory test } \\
\hline Immediate recall & $34 / 75^{*}$ \\
\hline Delayed recall & $8 / 15$ \\
\hline Recall of Rey-Osterrieth complex figure & $10,5 / 36^{*}$ \\
\hline \multicolumn{2}{|l|}{ Visuospatial attention } \\
\hline \multicolumn{2}{|l|}{ Bells cancellation test } \\
\hline Total misses & $9 / 35^{*}$ \\
\hline Center of cancellation & -0.002 \\
\hline \multicolumn{2}{|l|}{ Single letter cancellation test } \\
\hline Total misses & $5 / 104^{*}$ \\
\hline Center of cancellation & -0.008 \\
\hline \multicolumn{2}{|l|}{ Apples cancellation test } \\
\hline Total misses & $4 / 50$ \\
\hline \multicolumn{2}{|l|}{ Executive function } \\
\hline Letter fluency & $22^{*}$ \\
\hline Category fluency & 15,86 \\
\hline \multicolumn{2}{|l|}{ Trail making test } \\
\hline$A, \mathrm{~s}$ & $140^{*}$ \\
\hline$B, \mathrm{~s}$ & $320^{*}$ \\
\hline$B-A, \mathrm{~s}$ & $180^{*}$ \\
\hline
\end{tabular}

The asterisks indicate pathological scores based on the standardization and normative data obtained in the Italian population.

fMRI data averaged over the brain [9]. The DVARS criterion for high motion frames was set at 0.2 root mean square functional MRI signal change in units of \%. This frame-censoring criterion was uniformly applied to all resting-state fMRI data.

\section{DAN, Frontal Executive-Control, and LNs}

In the current study, we investigated the FC (see next paragraph) derived from a set of regions of interests (ROIs) belonging to the DAN (Fig. 3A) and the fECN (Fig. 3B). Moreover, we employed the LN as network of no-interest (see Table 2 for the complete list of ROIs, region label, and Talairach coordinates) [8]. 


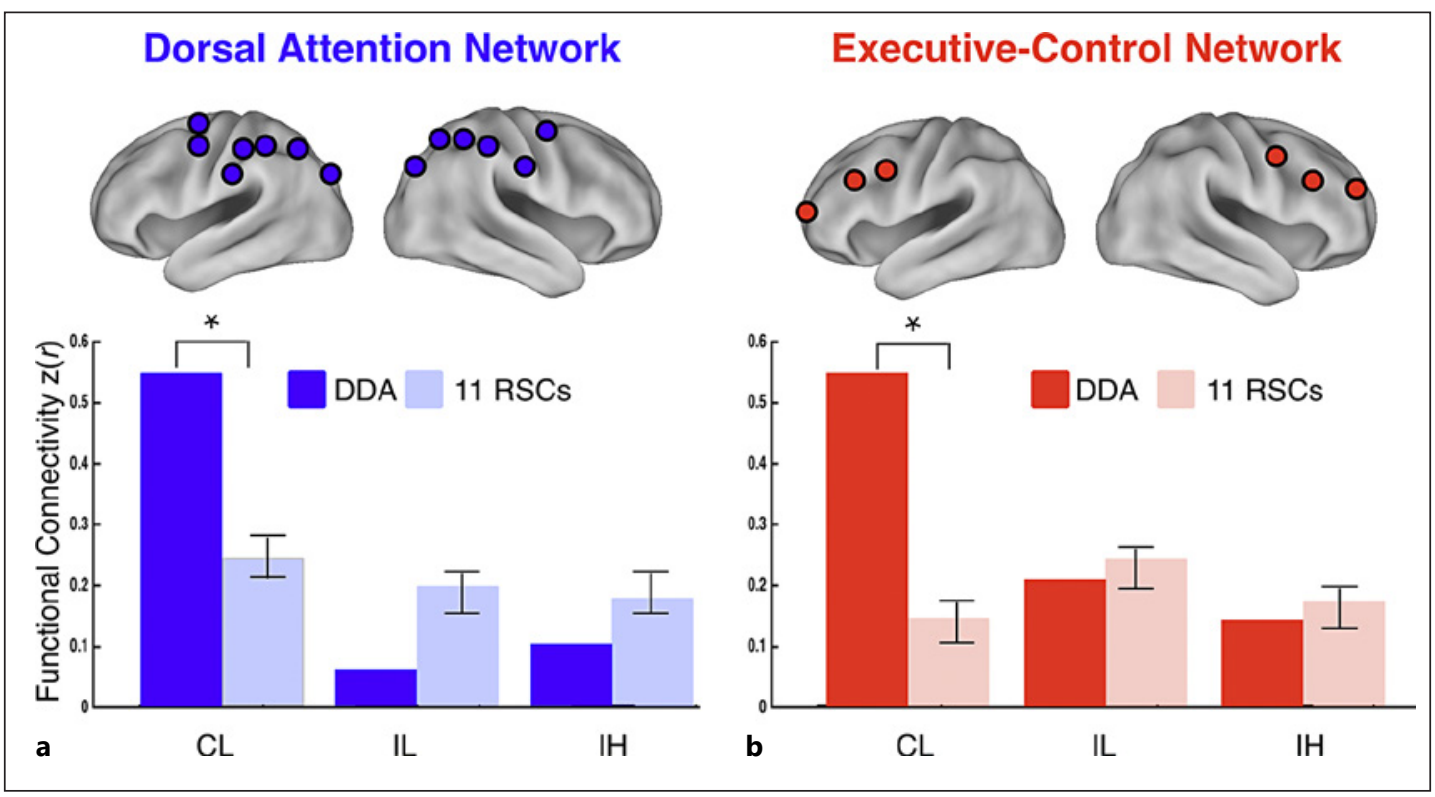

Fig. 3. Resting-state FC derived from DAN (A) and executive-control (B) computed in patient DDA (dark color bars) and 11 RSCs (light color bars). ${ }^{*} p<0.05$. CL, contralesional intra-hemispheric; IL, ipsilesional intra-hemispheric; IH, interhemispheric; FC, functional connectivity; DAN, dorsal attention network; RSC, right stroke control.

\section{Computing Resting-State FC}

The resting-state FC analyses included 3 steps.

1. Pair-wise FC. For patient DDA and each control patient, the resting-state FC was computed by extracting BOLD time courses from all ROIs and then correlating them (using $r$ Pearson) in a pairwise manner with the ones derived from all other ROIs. The $r$ scores were then transformed into $z$ values using the Fisher transform that was well-modeled by a normal distribution.

2. Network-wise FC. Based on the pair-wise FC values, for each network, we computed a score for 3 types of FC: (i) interhemispheric FC, by averaging the connectivity scores between each ROI and all other ROIs in the same network in the opposite hemisphere; (ii) ipsilesional intra-hemispheric FC, by averaging the connectivity scores between each ROI in the damaged (i.e., right) hemisphere and all other ROIs in the same network within the damaged hemisphere; and (iii) contralesional intra-hemispheric FC, same as the ipsilesional score but all ROI pairs were in the undamaged (i.e., left) hemisphere.

3. Comparison between DDA and stroke controls. For a given network, for example, DAN and FC type, for example, interhemispheric, we investigated whether the patient DDA, as compared to control group, exhibited a significant lower (or greater) FC score. To this aim, we computed the normal cumulative distribution function (normcdf) and its complementary (1-normcdf) corresponding to the probability that the distribution of the control group takes a value below (left tail area) or above (right tail area) the DDA's FC value, respectively. If the left (or right) tail area is $<0.025$, then the FC of single patient is significantly ( $p<0.05$ ) lower (or higher) than the control group. Finally, for each comparison, the $p$ value of the normcdf test was corrected for false discovery rate (FDR) at $\delta=0.05$. 
Table 2. Table lists all ROIs sorted by resting-state networks

\begin{tabular}{|c|c|c|c|c|c|}
\hline RSN & Hemisphere & Region & $\mathrm{X}$ & $Y$ & $Z$ \\
\hline DAN & Left & FEF & -19 & -8 & 57 \\
\hline DAN & Left & $\mathrm{dPrCe}$ & -27 & -10 & 47 \\
\hline DAN & Left & vPoCe-SMG & -53 & -29 & 37 \\
\hline DAN & Left & dPoCe & -45 & -34 & 45 \\
\hline DAN & Left & aIPS & -32 & -42 & 45 \\
\hline DAN & Left & mIPS & -22 & -53 & 52 \\
\hline DAN & Left & pIPS-SPL & -16 & -65 & 49 \\
\hline DAN & Right & $\mathrm{PrCe}$ & 45 & -3 & 34 \\
\hline DAN & Right & $\mathrm{FEF}$ & 23 & -8 & 55 \\
\hline DAN & Right & vPoCe-SMG & 53 & -28 & 36 \\
\hline DAN & Right & $\mathrm{dPoCe}$ & 46 & -32 & 50 \\
\hline DAN & Right & mIPS & 28 & -49 & 52 \\
\hline DAN & Right & pIPS-SPLd & 18 & -59 & 53 \\
\hline DAN & Right & pIPS-SPL & 20 & -67 & 43 \\
\hline DAN & Right & vIPSd & 26 & -69 & 30 \\
\hline DAN & Right & vIPS & 35 & -76 & 23 \\
\hline fECN & Left & aPFC & -28 & 51 & 15 \\
\hline fECN & Left & dlPFC & -43 & 22 & 34 \\
\hline fECN & Left & $\mathrm{dPrCe}$ & -41 & 3 & 36 \\
\hline $\mathrm{fECN}$ & Right & aPFC & 27 & 50 & 23 \\
\hline fECN & Right & dlPFC & 43 & 22 & 34 \\
\hline fECN & Right & $\mathrm{dPrCe}$ & 39 & 1 & 42 \\
\hline $\mathrm{LN}$ & Left & IFG & -48 & 30 & -2 \\
\hline LN & Left & IFG & -44 & 25 & -2 \\
\hline LN & Left & STG & -54 & -23 & -3 \\
\hline $\mathrm{LN}$ & Left & STG & -56 & -33 & 3 \\
\hline $\mathrm{LN}$ & Right & IFG & 47 & 25 & -4 \\
\hline LN & Right & IFG & 53 & 23 & 7 \\
\hline LN & Right & STG & 44 & -36 & 6 \\
\hline LN & Right & STG & 61 & -43 & 8 \\
\hline
\end{tabular}

RSN, resting-state network; DAN, dorsal attention network; fECN, frontal portion of executive-control network; LN, language network; FEF, frontal eye fields; dPrCe, dorsal precentral gyrus; vPoCe-SMG, ventral postcental gyrus-supramarginal gyrus; dPoCe, dorsal postcentral gyrus; aIPS, mIPS, pIPS, anterior, middle, posterior intraparietal sulcus, respectively; pIPS-SPL(d), posterior intraparietal sulcus-superior parietal lobule (dorsal); aPFC, anterior prefrontal cortex; dlPFC, dorsolateral prefrontal cortex; dPrCe, dorsal precentral gyrus; IFG, inferior frontal gyrus; STG, superior temporal gyrus. Talairach space; ROI, regions of interest.

\section{Results}

\section{Neuropsychological Profile of Patient DDA}

The neuropsychological assessment revealed a preserved general cognitive efficiency. Critically, DDA exhibited a constructional impairment as indicated by pathological score at the ROCF (Table 1) which was reproduced rotated along the vertical axis of $90^{\circ}$ both on copy 
and recall (Fig. 2). Moreover, DDA exhibited deficits of visual attention as indicated by a high number of total targets missed in the cancellation tests (Bells and Letter), long reaction times in the parts A and B of the Trail Making Test as well as poor performance in Corsi's Block Tapping Test. However, DDA did not exhibit signs of rightward spatial bias (i.e., visual neglect), since the scores of the Center of Cancellation on the cancellation tests were above the neglect cutoff. Accordingly, it can be ruled out that deficits of visual attention might be driven by visual neglect. Furthermore, deficits of executive functions were observed as shown by pathological scores of part B minus A of Trail Making Test and Phonemic Fluency test (Table 1). Finally, DDA presented deficits of both short (Corsi's Block Tapping Test) and long-term (ROCF, recall) spatial memory, whereas the verbal long-term memory was impaired for the immediate recall of unrelated items, but not for organized verbal material such as a story. In the control group, it should be considered that for each domain the cohort is different as some patients did not carry out the tests for several reasons such as fatigue or lack of collaboration, thus the results are reported at a descriptive level (see online suppl. Table 1; for all online suppl. material, see www.karger.com/doi/10.1159/000518844).

\section{Hyper-Connectivity of the Undamaged DAN and fECN in Patient DDA}

Based on the computation of the complementary normcdf on the FC scores derived from the regions of the DAN, we observed that DDA patient $(z=0.61)$, as compared to the right stroke control patients (RSCs) (mean $z=0.34$; SE $=0.03$ ), exhibited significantly higher contralesional intra-hemispheric FC (normcdf test, $p=0.006$, FDR-corrected at $\delta=0.05$ ) (Fig. 3A), whereas no differences were observed for the interhemispheric (DDA, $z=0.11$; RSCs, $z=0.16$; SE $=0.03$; normcdf test $p=0.28$ ) and ipsilesional intra-hemispheric (DDA, $z=$ 0.12 ; RSCs, $z=0.31$; $\mathrm{SE}=0.02$; normcdf test $p=0.01$ not surviving FDR correction at $\delta=0.05$ ) FC scores. Furthermore, a similar pattern was found for the FC scores obtained from fECN as DDA patient, as compared to stroke controls, selectively showed significant higher contralesional intra-hemispheric FC (DDA, $z=0.7$; RSCs, $z=0.43$; SE $=0.03$; normcdf test $p=0.003$, FDR-corrected at $\delta=0.05$ ) (Fig. 3B) but not ipsilesional intra-hemispheric FC (DDA, $z=0.46$; RSCs, $z=0.49 ; \mathrm{SE}=0.04$; normcdf test $p=0.57$ ) and inter-hemispheric FC (DDA, $z=0.13$; RSCs, $z=0.16 ;$ SE $=0.04$; normcdf test $p=0.59$ ).

Moreover, to link DDA's pattern of FC to drawing rotation, we identified a control patient (FG) (M, 67-year-old, 13-year education) with a comparable neuropsychological profile as DDA, showing a pathological scoring on Corsi's Block Tapping test (raw score $=2$ ), TMT (raw scores, $A=80 \mathrm{~s} ; B=286 \mathrm{~s} ; A-B=206 \mathrm{~s}$ ), Bells cancellation test (total misses =13) and copy of ROCF (raw score $=30$ ), without rotated drawing. At descriptive level, patient DDA exhibited higher left intra-hemispheric FC of DAN and fECN, as compared to control patient FG (DDA, $z$ $=0.61 ; \mathrm{FG}, z=0.464$ for left DAN; DDA, $z=0.61 ; \mathrm{FG}, z=0.51$ for left fECN). Notably, the computation of the complementary normcdf indicated that left DAN FC of FG patient $(z=0.464)$ did not statistically differ from the one of the RSC patients $(n=10$; mean $z=0.33$; SE $=0.032$ ) (normcdf test, $p=0.1$ ). A similar pattern was observed for the left intra-hemispheric FC of fECN when comparing patient FG $(z=0.51)$ with the RSCs $(n=10$; mean $z=0.43$; SE $=0.031)$ (normcdf test, $p=0.21$ ).

Finally, to test whether the association between rotated drawing and the FC pattern was specific for DAN and fECN, we repeated the same analyses on the FC scores derived from language network, which is not supposed to play a central role in copy and recall a complex figure. Crucially, none of DDA's FC scores obtained from LN was significantly different from the ones of the control patients (contra-lesional FC: DDA, $z=0.48$; RSCs, $z=$ 0.47 ; SE $=0.04$; normcdf test $p=0.48$; interhemispheric FC: DDA, $z=0.23$; RSCs, $z=0.14$; $\mathrm{SE}=0.02$; normcdf test $p=0.14$; ipsilesional FC: DDA, $z=0.35$; RSCs, $z=0.39 ; \mathrm{SE}=0.02$; normcdf test $p=0.64$ ).

\section{Karger'}




\section{Discussion}

In the present case study, we investigated the neuropsychological profile and brain network functional architecture of a patient (DDA) who produced rotated drawing of the Rey Complex Figure at copy and recall. Behaviorally, beside impairments of constructional abilities, the neuropsychological profile comprised deficits of visual attention and executive functions. These patterns are in line with previous reports on patients with degenerative disorders and Mild Cognitive Impairment [2, 3]. Critically, patient DDA exhibited network-specific hyper-connectivity of the undamaged (i.e., left) hemisphere within the DAN (i) and frontoparietal executive-control (ii) networks. Noteworthy, such neurofunctional patterns were not detected in a control patient showing a neuropsychological profile overlapping with the one of DDA, yet without rotated drawing. Hence, it can be argued that the increased FC can be selectively associated with the constructional phenomenon of rotated drawing, rather than to spatial and visuomotor deficits. Such pattern might reflect the unbalanced activations between damaged and healthy hemispheres widely observed after focal lesion [10], consistently with a pathological modulation of these networks during attentional and executive operations required to reproduce the model in the correct orientation. Specifically, given the role of DAN in the control of visuospatial attention [4], the increased connectivity detected between its regions could be associated with the impairments of several abilities required for analyzing the spatial properties of the stimuli and for reproducing the model in the correct orientation [11]. At the same time, the pattern involving the fECN might be related to the dys-executive deficits of planning and monitoring processes which are required to accomplish the current task [1]. Finally, a not-exclusive possibility is that the concomitant hyper-connectivity of DAN and fECN could reflect the DDA's distractibility, which might impair both attentive and executive processes engaged during the steps of drawings on copy and memory.

From a theoretical point of view, the current findings represent a clear example of "connectomal diaschisis" [12] as a focal lesion has induced wide-spread behaviorally relevant changes of the FC within brain areas which are distant from the site of the stroke [13]. This is in line with noninvasive brain stimulation studies in healthy subjects showing that interference of a crucial area produces impairment of behavior [14] and neurophysiological markers [15] not limited to the targeted cortical region, but also to its functionally connected areas. Clinically, these findings might be useful for planning the rehabilitation of patients suffering of such constructional impairment by employing Transcranial Magnetic Stimulation over brain networks whose FC was pathologically increased.

\section{Limitations}

The case report presents some limitations. First, DDA exhibited several behavioral impairments; therefore, it might be possible that other deficits can contribute to the abnormal pattern of FC. Second, our analyses were restricted to the FC within DAN and FECN, thus further investigations should explore the interaction between these networks. Finally, DDA was studied at the acute stage and follow-up protocols might be useful for understanding the neural signature of the recovery of such phenomenon.

\section{Statement of Ethics}

Written informed consent was obtained from patient DDA and all participants for publication of this case report and any accompanying images. The research was approved by the Institutional Review Board of NEUROMED, protocol number 10/16, on July 28, 2016.

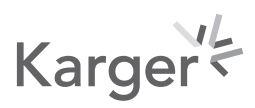




\section{Conflict of Interest Statement}

The authors have no conflict of interest or disclosure to be reported.

\section{Funding Sources}

This work was supported by the Ministry of Health Italy.

\section{Authors Contributions}

V.S. and A.B. contributed conception and design of the study; L.P. and A.B. collected data; V.S., P.C., L.P., and A.B. organized the database; V.S., P.C., S.S., A.S., G.G., and A.B. performed the statistical analysis; V.S., P.C., G.C., and A.B. wrote sections of the manuscript. All the authors contributed to manuscript revision, read, and approved the submitted version.

\section{Data Availability Statement}

The raw data supporting the conclusions of this manuscript will be made available by the authors, without undue reservation, to any qualified researcher.

\section{References}

1 Solms M, Turnbull OH, Kaplan-Solms K, Miller P. Rotated drawing: the range of performance and anatomical correlates in a series of 16 patients. Brain Cogn. 1998 Dec;38(3):358-68. PubMed PMID: 9841791.

2 Isella V, Traficante D, Tagliabue CF, Mapelli C, Ferri F, Caffarra P, et al. A retrospective survey on rotated drawing in persons with mild cognitive impairment or degenerative dementia. Clin Neuropsychol. 2013; 27(8):1300-15. PubMed PMID: 24099048.

3 Molteni F, Traficante D, Ferri F, Isella V. Cognitive profile of patients with rotated drawing at copy or recall: a controlled group study. Brain Cogn. 2014 Mar;85:286-90. PubMed PMID: 24509088.

4 Corbetta M, Shulman GL. Control of goal-directed and stimulus-driven attention in the brain. Nat Rev Neurosci. 2002 Mar;3(3):201-15. PubMed PMID: 11994752.

5 Fox MD, Raichle ME. Spontaneous fluctuations in brain activity observed with functional magnetic resonance imaging. Nat Rev Neurosci. 2007 Sep;8(9):700-11. PubMed PMID: 17704812.

6 Seeley WW, Menon V, Schatzberg AF, Keller J, Glover GH, Kenna H, et al. Dissociable intrinsic connectivity networks for salience processing and executive control. J Neurosci. 2007 Feb 28;27(9):2349-56. PubMed PMID: 17329432.

7 Dosenbach NU, Fair DA, Miezin FM, Cohen AL, Wenger KK, Dosenbach RA, et al. Distinct brain networks for adaptive and stable task control in humans. Proc Natl Acad Sci U S A. 2007 Jun 26;104(26):11073-8. PubMed PMID: 17576922.

8 Baldassarre A, Ramsey L, Hacker CL, Callejas A, Astafiev SV, Metcalf NV, et al. Large-scale changes in network interactions as a physiological signature of spatial neglect. Brain. 2014 Dec;137(Pt 12):3267-83. PubMed PMID: 25367028. Pubmed Central PMCID: 4240302.

9 Power JD, Barnes KA, Snyder AZ, Schlaggar BL, Petersen SE. Spurious but systematic correlations in functional connectivity MRI networks arise from subject motion. Neuroimage. 2012 Feb 1;59(3):2142-54. PubMed PMID: 22019881. Pubmed Central PMCID: 3254728. Epub 2011/10/25. eng.

10 Corbetta M, Kincade MJ, Lewis C, Snyder AZ, Sapir A. Neural basis and recovery of spatial attention deficits in spatial neglect. Nat Neurosci. 2005 Nov;8(11):1603-10. PubMed PMID: 16234807.

11 Trojano L, Conson M. Visuospatial and visuoconstructive deficits. Handb Clin Neurol. 2008;88:373-91. PubMed PMID: 18631702.

12 Carrera E, Tononi G. Diaschisis: past, present, future. Brain. 2014 Sep;137(Pt 9):2408-22. PubMed PMID: 24871646.

13 Baldassarre A, Metcalf NV, Shulman GL, Corbetta M. Brain networks' functional connectivity separates aphasic deficits in stroke. Neurology. 2019 Jan 8;92(2):e125-35. PubMed PMID: 30518552 
Case Reports in Neurology

14 Baldassarre A, Capotosto P, Committeri G, Corbetta M. Magnetic stimulation of visual cortex impairs perceptual learning. Neuroimage. 2016 Dec;143:250-5. PubMed PMID: 27591920.

15 Croce P, Spadone S, Zappasodi F, Baldassarre A, Capotosto P. rTMS affects EEG microstates dynamic during evoked activity. Cortex. 2021 Mar 4;138:302-10. 